\title{
Cirurgião Oncológico, fator de prognóstico no tratamento do câncer
}

\author{
Oncologic Surgeon: a prognostic factor in the treatment of cancer
}

Odilon de Souza Filho ${ }^{1}$

$\mathrm{H}$ á alguns anos a cirurgia oncológica vem sendo reconhecida como uma especialidade cirúrgica com importante papel no tratamento de câncer. 0 cirurgião oncológico atua de várias maneiras: como consultor; na realização de cirurgias simples e complexas; nos programas de avaliação; como organizador e líder de serviços cirúrgicos; na elaboração de programas de câncer; participando dos comitês de câncer; na criação de registro de câncer; nos serviços de oncologia, atuando como professor; e finalmente trabalhando como investigador, elaborando e executando protocolos clínicos. Há inúmeros motivos para evolução desta especialidade e os mais significativos são a complexidade crescente de cuidados e possibilidades terapêuticas multidisciplinares para o câncer, as oportunidades de investigações clinicas e laboratoriais relacionadas à biologia tumoral e a expectativa que o cirurgião tem em relação às mais recentes informações e novas opções de tratamento.

H á algum tempo, autores 1,2,3,4,5,6,7,8,9,10,11 vêm publicando artigos demonstrando fatores que alteram 0 prognóstico de um tratamento. Os fatores de prognósticos mais utilizados são: o tamanho de tumor ou o comprometimento do tumor no órgão, extensão do envolvimento linfonodal, presença de metástase à distância, invasão vascular, invasão neural, diferenciação celular. 0 cirurgião, dentre os vários fatores que influenciam o resultado do tratamento, é considerado uma variável importante no tratamento do câncer, pois por sua atuação será alterada a evolução dos casos e influenciadas a mortalidade pós-operatória, morbidade pós-operatória, sobrevida, intervalo livre de doença, taxa de recidiva e qualidade de sobrevida. Estas variáveis estão relacionadas diretamente com o volume de casos tratados e o treinamento prévio do cirurgião (curva de aprendizado). 5,9,10,11,12,13.14,15

Um estudo realizado no Canadá evidenciou taxa de mortalidade pós-operatória de $22 \%$ quando a cirurgia foi executada por cirurgiões que realizaram menos de 6 esofagectomias/ano e mortalidade de $0 \%$ para os cirurgiões que realizaram mais de seis casos por ano.2 Begg et al.,12 utilizando o banco de dados do M edicare, analisaram o impacto da mortalidade pós-esofagectomia, pancreatectomia e exenteração pélvica em função do o volume de casos operado. O bservaram que num hospital que trata um maior volume de pacientes com procedimentos cirúrgicos oncológicos apresenta uma baixa taxa de mortalidade comparado com hospitais que têm um menor volume de procedimentos. Estes dados confirmam a hipótese que nos procedimentos cirúrgicos oncológicos complexos, quando realizados por uma equipe cirúrgica experiente e em hospital especializado, as taxas de mortalidade são mais baixas.

A taxa de recidiva éum grande indicador de eficiência no tratamento oncológico. $\mathrm{H}$ eald et al., ${ }^{5}$ através da técnica de excisão completa do mesorreto no tratamento cirúrgico dos tumores do reto, obteve taxa de recidiva de $6 \%$, e a literatura mostra uma taxa que varia de $20 \%$ a 35\% 6,16 após a ressecção clássica. M cArdle, ${ }^{7}$ analisando o câncer colorretal, notou diferenças importantes entre cirurgiões quando examinou a morbidade pósoperatória, mortalidade pós-operatória e sobrevida do paciente. As taxas de ressecções curativas variaram de $40 \%$ a $76 \%$, mortalidade de $8 \%$ a $30 \%$, recidiva local de 0 a 21\%, recidiva na região da anastomose de 0 a $25 \%$, e a sobrevida após 10 anos de ressecção curativa de $20 \%$ a $63 \%$. Gillis 8 observou semelhantes variações para o câncer da mama.

Tais variações são ilustradas bem por M cArdle e $\mathrm{H}$ ole $\mathrm{e}^{12}$ em estudo realizado na Escócia, onde foi

${ }_{1}^{1}$ Presidente da Sociedade Brasileira de Cirurgia O ncológica 
observado que a sobrevida no tratamento cirúrgico de câncer colorretal tem como fator de risco o cirurgião, com uma variação de 0,56 a 2,03, e que dependendo do cirurgião, há uma diferença de quatro vezes no risco de morte em 5 anos.

0 volume de casos por hospital também parece ser uma importante variável. N os tumores do pâncreas, há uma correlação direta entre volume de casos de um hospital e a mortalidade pós-operatória.,13 U m número maior de casos por instituição, isto é, sua centralização em um hospital especializado, permite realizar protocolos e desenvolver diretrizes de tratamento, possibilitando assim uma melhor solução nas complicações. Concluímos que, "cirurgiões eventuais ou esporádicos" deveriam evitar executar procedimentos cirúrgicos complexos e sim encaminhar seus pacientes a centros de excelência ou a cirurgiões com maior experiência na patologia.

A eficiência do resultado do tratamento cirúrgico do câncer está, em muito, relacionada ao preparo técnicopessoal, treinamento específico, volume de casos e ao interesse que o profissional desenvolve em relação à patologia.

O caracter multidisciplinar está diretamente relacionado à infra-estrutura específica do hospital. Van Lanschot ${ }^{11}$ analisou a mortalidade hospitalar de esofagectomia por câncer na H olanda entre 1993 e 1998. H ospitais com menos que 10 casos por ano tinham $12 \%$ de mortalidade hospitalar. H ospitais com mais que 30 ressecções por ano tinham uma taxa de mortalidade hospitalar de 4,9\%. Romano e M ack estimaram que o risco de mortalidade em 30 dias após a ressecção de câncer do pulmão foi $40 \%$ menor nos centros com maior volume de atendimento do que nos de menor volume. ${ }^{14}$

Devemos ser rígidos no controle de qualidade nos programas de treinamento. A qualidade de treinamento cirúrgico é um fator importante que irá reduzir as variações nos resultados. $\mathrm{N}$ a literatura há dados suficientes de que um cirurgião, quando bem treinado e bem supervisionado obterá resultado semelhante ao do supervisor ou do cirurgião sênior, sem comprometer o resultado imediato quanto à sobrevida a longo prazo. ${ }^{17}$ o período de treinamento é, sem dúvida, a melhor oportunidade para influenciar favoravelmente a qualidade de qualquer futuro cirurgião oncológico.

0 cirurgião oncológico tem a responsabilidade de promover a saúde oncológica na área cirúrgica, sendo um diferencial construtivo na sobrevida dos pacientes e no enfoque de qualidade e reabilitação que o tratamento oncológico deve trazer consigo.

\section{REFERÊNCIAS BIBLIOGRÁFICAS}

1. M atthews HR, Powell DJ, M cC onkey CC. Effects of surgical experienceon theresults of resection for oesophageal carcinoma. Br J Surg 1986;73:621-3.

2. M iller JD, Jain M K, deG ara CJ, M organ D, U rschel JD. Effect of surgical experience on results of esophagectomy for esophageal carcinoma. J Surg 0 ncol 1997;65(1):20-1.

3. Andersen $K B, O I$ sen $J B$, Pedersen JJ. Esophageal resections in D enmark, 1985-1988: a retrospectivestudy of complications and early mortality. U geskr Laeger 1994;156:473-6.

4. Sowden AJ, D eeks JJ, Sheldon TA. Volumeand outcome in coronary artery bypass graft surgery: trueassociation or artifact. BMJ 1995;331:151-5.

5. H eald RJ, M oran BJ, Ryall RD, Sexton R, M acFarlaneJK. Rectal cancer: theBasingstokeexperience of total mesorectal excision 1978-1997. Arch Surg 1998;133(8):894-9.

6. H avenga $K$, Enker W E, N orstein J, M oriya Y, H eald RJ, van $\mathrm{H}$ ouwelingen $\mathrm{HC}$, et al. I mproved survival and local control after total mesorectal excision or D 3 lymphadenectomy in thetreatment of primary rectal cancer: an international analysis of 1411 patients. Eur J Surg O ncol 1999;25(4):368-74.

7. M cArdle CS, H ole D. I mpact of variability among surgeonson postoperativemorbidity and mortality and ultimate survival. BMJ 1991;302:1501-5.

8. Gillis CR, H oleD J. Survival outcome of care by special ist surgeons in breast cancer: a study of 3786 patients in the west of Scotland. BM J 1996;312:145-8.

9. N eoptolemos JP, Russell RC, Bramhall S, Theis B. Low mortality following resection for pancreatic and periampullary tumour of 1026 patients: U K survey of specialist pancreatic units. UK Pancreatic $C$ ancer $\mathrm{Group}$. $\mathrm{Br}$ J Surg 1997;84(10):1370-6.

10. Swisher SG , D eford L, M erriman KW, Walsh GL, Smythe $R$, Vaporicyan $A$, et al. Effect of operativevolume on morbidity, mortal ity and hospital useafter esophagectomy for cancer. J T horac C ardiovasc Surg 2000;119(6):1126-32.

11. van Lanschot JJ, Rutten HJ, Boom RP, G ouma DJ. Importance of regional surgery networks. $\mathrm{N}$ ed Tijdschr $\mathrm{G}$ eneeskd 2000;144(24):1148-52.

12. Begg CB, Cramer LD , H oskinsWJ, Brennan M F. Impact of hospital volumeon operativemortality for major cancer surgery. JAM A 1998;280:1747-51.

13. GoumaD J, van Geenen RC, van GulikTM, deH aan RJ, de W it LT, Busch OR, et al. Rates of complications and death after pancreaticoduodenectomy: risk factors and theimpact of hospital volume. Ann Surg 2000;232(6):786-95.

14. Romano PS, M ack D H . Patient and hospital characteristics related to in-hospital mortality after lung cancer resection. Chest 1992;101:1332-7.

15. Stockholm Colorectal C ancer Study G roup. Randomized study on preoperative radiotherapy in rectal carcinoma. 
Ann Surg O ncol 1996;3:423-30.

16. Bonenkamp JJ, H ermans], Sasako M, van deVeldeCJ. Extended lymph-nodedissection for gastric cancer. D utch Gas- tric Cancer Group. N Engl J M ed 1999;340(12):908-14.

17. Sutton D N, Wayman J, G riffin SM . Learning curve for oesophageal cancer surgery. BrJ Surg 1998;85:1399-402. 